\title{
Effect of Ubiquinol on Exercise and the Oxidative Stress Regulation System in SMAP1 Mice
}

\author{
Hiroshi Maruoka, PT, PhD ${ }^{1)}$, Kenji Fujit, PhD'2), Kazuhisa Inoue, $\mathrm{PT}^{1)}$ \\ 1) School of Health and Social Services, Saitama Prefectural University: 820 San-nomiya, Kosigaya, \\ Saitama, 343-8540, Japan. TEL: +81 48-973-0500, FAX: +81 48-973-4807, E-mail: maruoka- \\ hiroshi@spu.ac.jp \\ 2) Functional Food Ingredients Group, QOL Division, Kaneka Corporation
}

\begin{abstract}
Purpose] This study examined how exercise capacity and the oxidative stress regulation system are affected by different amounts of consumed ubiquinol (reduced coenzyme Q10, $\mathrm{H}_{2} \mathrm{CoQ} 10$ : QH) through experiments on 23 SAMP1 mice. [Methods] The mice were randomly divided into two groups: one consuming a high amount of $\mathrm{QH}(300 \mathrm{mg} / \mathrm{Kg})$ and the other consuming a low amount of QH $(30 \mathrm{mg} / \mathrm{Kg})$. Both groups were made to run up to their limit on a treadmill (TM) before/after consuming $\mathrm{QH}$, and then each running time was measured. For the oxidative stress regulation system, the d-ROM test value (degree of oxidative stress) and BAP test value (anti-oxidant potential) were measured both in a resting state before $\mathrm{QH}$ consumption and after running up to the limit, and then the BAP/d-ROM ratio was calculated. The values of plasma $\mathrm{QH}$ and plasma ubiquinone (plasma oxidized CoQ10) were also measured, and the reduced ratio was calculated. [Results] Both groups showed a significant extension of running time. Also, a more significant extension was seen in the group consuming a high amount of QH than in the group consuming a low amount. With regard to the oxidative stress regulation system, the group consuming a high amount also showed a significant increase in d-ROM test value, plasma $\mathrm{QH}$ value and reduced ratio. [Conclusion] The difference in the amount of consumed ubiquinol led to an extension of running time and an increase in reduced ratio and other values.
\end{abstract}

Key words: Oxidative stress regulation system, Reduced coenzyme Q10 $\left(\mathrm{H}_{2} \mathrm{CoQ} 10\right)$, Exercise

(This article was submitted Oct. 12, 2012, and was accepted Nov. 7, 2012)

\section{INTRODUCTION}

Oxygen is essential to maintain homeostatic function, but for some reason, some of it is changed into active oxygen (superoxide radical etc. is collectively referred to as oxidant stress), which damages body cells and tissues and causes various illnesses like lifestyle-related diseases ${ }^{1)}$. The body has various defensive mechanisms against this, including an autonomic system, an endocrine system, an immune system and an oxidative stress regulation system ${ }^{2}$. In particular, the oxidative stress regulation system ensures a "balance (latent anti-oxidant potential) between the oxidative reaction (oxidative stress) and anti-oxidant reaction (anti-oxidant potential) of the body," and how the defensive capability against oxidative stresses (anti-oxidant potential) can be improved is important. As oxygen flux to tissues increases during exercise ${ }^{3)}$, the amount of oxidant stress also shows a remarkable rise. How transient or continuous exercise loaded on mice has an impact on oxidative stress and anti-oxidant potential has already been reviewed ${ }^{4}$, but there have been few studies on the relation between exercise capacity and the oxidative stress regulation system through consumption of vitamin $\mathrm{C}$, which is sensitive to oxidative stress, and antioxidant foods containing a radical scavenger like ubiquinol (reduced coenzyme Q10, $\left.\mathrm{H}_{2} \mathrm{CoQ} 10: \mathrm{QH}\right)^{5,}$ ). In addition, there have been no studies on how different amounts of consumed antioxidant foods affect exercise capacity and degree of oxidative stress. Like cholesterol, Coenzyme Q (CoQ), is biologically synthesized in the mevalonate pathway and exists in all organs and cells ${ }^{7}$. Coenzyme $Q$ has two major functions: activating energy production and antioxidation. A major CoQ in mammals like humans is CoQ10, which has a side chain with ten isoprenoid units, existing as ubiquinone (oxidized CoQ10) and ubiquinol. CoQ10 is converted by reductase from an oxidized form to a reduced form in the body with $\mathrm{NAD}(\mathrm{P}) \mathrm{H}$ serving as an electron donor, and the conversion capability decreases with age ${ }^{8)}$. While it has been reported that CoQ10 consumption by mice leads to an increase in plasma CoQ10 and other values ${ }^{7,9)}$, we have not seen a report that studied how different amounts of consumed antioxidant foods affect the reduced ratio.

Here, we studied the effect of different amounts of $\mathrm{QH}$ consumed by SAMP1 mice(SAMP1/Sku Slc: SAM) on exercise capacity and the oxidative stress regulation system, such as the degree of oxidative stress.

\section{SUBJECTS AND METHODS}

Our study was performed on 23 male SAM (38 weeks old), which were randomly divided into two groups: one 
consuming a high amount of $\mathrm{QH}(300 \mathrm{mg} / \mathrm{Kg}, \mathrm{n}=12)$ and the other consuming a low amount of $\mathrm{QH}(30 \mathrm{mg} / \mathrm{Kg}$, $\mathrm{n}=11$ ). At 38 weeks of age, all the mice were made to perform running exercise two times to get acclimated to the treadmill (model TM-R-N1, Osakamicro, Japan: TM) (I, speed $20 \mathrm{~m} / \mathrm{min}$, tilt 0 degrees, time $30 \mathrm{~min}$; II, speed $20 \mathrm{~m} / \mathrm{min}$, tilt 10 degrees, time $30 \mathrm{~min}$ ). The first running exercise (I) was done five days before the measurement of running time, and the second exercise (II) was performed four days before measurement. All of the SAMP1 mice were kept at room temperature $\left(20 \pm 1{ }^{\circ} \mathrm{C}\right)$, at a relative humidity of about $50 \%$ and with a light-dark cycle of 12 hours; food (CE-2, CLEA Japan, Inc.) and tap water were provided ad libitum. The SAM used in this study was a useful model in that senescence was accelerated after it grew up, and it was assumed to live for $50 \%$ of its survival time (297 days on average $)^{10,11)}$. In this study, running time (exercise capacity), the oxidative stress regulation system, senescence grading scores and weight were measured at 38 to 39 weeks of age. The two groups were again (at 39 weeks of age), made to run on the TM up to their limit two days before $\mathrm{QH}$ consumption (running condition I) and three hours after $\mathrm{QH}$ consumption (running condition II), and the running time was regarded as their exercise capacity ${ }^{6}$. The time set for after QH consumption was based on our previous study, in which oxidized CoQ10 was orally administered to mice ${ }^{12)}$. A treadmill with its speed set to $25 \mathrm{~m} / \mathrm{min}$ and its tilt set at 20 degrees was used for the exercise load. The termination criterion for the limit of running was decided as the point when the time interval of electrostimulation delivered to the back of the running surface of the treadmill fell below 5 seconds ${ }^{6}$. The amount of $\mathrm{QH}$ consumption was based on our preceding study ${ }^{9}$, and a previous study reported no problems in a safety test in which mice consumed $1200 \mathrm{mg}$ / $\mathrm{kg}$ /day at maximum for 13 weeks $^{13)}$. Using analytical equipment for reactive oxygen and free radicals (model FRAS4, H\&D, Parma, Italy), the d-ROM test value (reactive oxygen metabolites test: degree of oxidative stress) and BAP test value (biological anti-oxidant potential) were measured in a resting state at the age of 38 weeks (before acclimation to running exercise on the $\mathrm{TM}$ before $\mathrm{QH}$ consumption) and right after running under condition II (right after running to the limit after $\mathrm{QH}$ consumption), and then latent antioxidant potential (BAP/d-ROM ratio) was calculated. As an oxidative stress marker, the values of plasma $\mathrm{QH}$ and plasma ubiquinone (plasma oxidized CoQ10, plasma Q10) were measured using an electrochemical detector (Shiseido Co., Ltd., Tokyo, Japan), and the reduced ratio (plasma QH value / plasma $\mathrm{QH}$ value + plasma Q10 value) was calculated. For the d-ROM and BAP measurements, blood was extracted from the tail vein and centrifuged immediately (for five minutes at 6,000 rpm) to obtain blood plasma for analysis. In the d-ROM test, the levels of free radicals in the body, especially hydroperoxide concentrations, were measured (unit: U.CARR, 1 U.CARR $=0.08 \mathrm{bmg} / \mathrm{dl}$ of hydrogen peroxide) according to the optical measurement method (color reaction), and the measured value indicates the degree of oxidative stress (oxidative reaction) ${ }^{14,15}$ ) Meanwhile, in the BAP test, the levels were measured (unit: $\mu \mathrm{M})$ by the reduction action of anti-oxidant materials in blood plasma, and the measured values indicate the degree of anti-oxidant potential (anti-oxidant reaction). That is, the amount of blood plasma that, when mixed with reagents containing ferric ions was reduced to ferrous ions, was measured at the decoloring level of the color reaction liquid according to the optical measurement method. The content of iron ions to which blood plasma is reduced is the antioxidant potential ${ }^{14,15}$ ). The BAP/d-ROM ratio, which was calculated on the basis of the values obtained in the BAP test and d-ROM test, indicates the degree of latent anti-oxidant potential. In other words, the latent anti-oxidant potential indicates the balance between oxidative stress and antioxidant potential. Senescence grading scores, which were designed to evaluate senescence more objectively, were graded according to changes in hair luster, lesions around eyes, bend of the spine and so on ${ }^{10)}$. Senescence grading scores are generally used as an acceptance criterion for the aging process. Higher scores mean that the aging process is accelerated. In the present study, we determined the grade of senescence, taking our preceding study into account ${ }^{10)}$. Weight was measured in a resting state at the age of 38 weeks using scales for animals (KN type manufactured by Natsume Corporation). Numerical values are presented in this study as average values \pm standard deviation. SPSS (Ver 19.0 for win) was used as for statistical analyses, and the Mann-Whitney U test was used to test the significance of the differences. This study was conducted with the approval of the Animal Research Committee of Saitama Prefectural University (approval number: 32).

\section{RESULTS}

As a result of $\mathrm{QH}$ consumption, both groups showed a significant extension of running time $(\mathrm{p}<0.05)$ (Table 1$)$. Also, a more significant extension was seen in the group consuming a high amount of QH than in the group consuming a low amount $(\mathrm{p}<0.05)$. Only the group consuming a high amount of QH showed a significant increase in d-ROM test value after $\mathrm{QH}$ consumption in comparison with the value before $\mathrm{QH}$ consumption $(\mathrm{p}<0.01)$, and both groups showed a significant increase in BAP test value and BAP/d-ROM ratio $(p<0.01)($ Table 2$)$. Comparison of the $d-R O M$ test values of the groups showed a more significant increase after $\mathrm{QH}$ consumption in the group consuming a high amount $(\mathrm{p}<0.05)$. This group showed a significant increase after $\mathrm{QH}$ consumption in the values of plasma $\mathrm{QH}$ and plasma Q10 and a reduced ratio, compared with the values before QH consumption $(\mathrm{p}<0.01-0.001)$, while the group consuming a low amount showed a significant decrease in plasma oxidized Q10 and a significant increase in reduced ratio $(p<0.05, p<0.01)$ (Table 3$)$. In particular, the value of plasma $\mathrm{QH}$ in the group consuming a high amount showed a nearly tenfold increase. Comparison of plasma $\mathrm{QH}$, plasma Q10 and the reduced ratio showed a significant increase after $\mathrm{QH}$ consumption in the group consuming a high amount $(\mathrm{p}<0.001$ each). The average senescence grading score was $2.6 \pm 0.7$ in the group consuming a high amount of $\mathrm{QH}$ and $2.4 \pm 0.4$ in the group consuming a low amount. The 
average weight was $35.7 \pm 2.2 \mathrm{~g}$ in the group consuming a high amount and $33.4 \pm 2.6 \mathrm{~g}$ in the group consuming a low amount. These values did not reveal any differences between the groups.

\section{DISCUSSION}

The purpose of this study was to examine the effects of different amounts of consumed QH on exercise capacity and the oxidative stress regulation system. In particular, QH is a typical supplement material, attracting attention for activating energy production and having anti-oxidation properties. Although its biological effect has been demonstrated $\left.{ }^{7}, 16-18\right)$, it has not been revealed how exercise capacity and the oxidative stress regulation system are affected by different amounts of consumed QH. Thus, our aim was for this to be a fundamental study for consideration of the oxidative stress system and examination of measures for improving exercise capacity. CoQ10 has an anti-fatigue action and has been used a lot in Europe and the United States for strengthening the physical aspects of athletes ${ }^{17)}$. Mizuno et al. ${ }^{19)}$ reported that the consumption of oxidized CoQ10 by healthy individuals was effective in relieving the physical aspects of fatigue resulting from ergometer exercise. Since it was revealed that $\mathrm{QH}$, when orally administered, was transformed into $\mathrm{QH}$ soon after it was absorbed in the small intestine, the above report essentially showed the effect of QH. In the present study, consumption of $\mathrm{QH}$ resulted in a significant extension of running time. This physiological effect on running time showed the same tendency as in our preceding study ${ }^{19}$ ) through the consumption of oxidized CoQ10. In other words, this effect was thought to have lasted not only because of the activation of mitochondria-enhanced myocardial metabolism (increase in cardiac output) but also because oxygen demand for active muscles was efficiently met by a decrease in peripheral vascular resistance (increase in oxygen avail- ability in the periphery). Even low consumption showed a physiological effect on running time. Moreover, the group consuming a high amount of QH in the present study showed a more significant extension of running time than the group consuming a low amount, which suggested the possibility that $\mathrm{QH}$ consumption caused the physiological effect to be prominent. The body's defense against oxidative lesions is not perfect, and oxidation products formed through exercise indicate oxidative stress ${ }^{5}$. In general, the effect of exercise on oxidative stress increases with the extension of running time, since oxygen is taken in into the body for the purpose of continuous production of $\mathrm{ATP}^{20}$. Exposure to such things as exercise is reported to have the possibility, potential to increase the expression of enzymes of the bio-defense system, including anti-oxidant enzymes, and proteins and control the expression of inflammation-related enzymes in response to enhanced production of oxidative stress ${ }^{21)}$. It has been pointed out that the activation of these anti-oxidant enzymes is a compensatory response to increased production of oxidative stress ${ }^{22}$. The body has a vitamin $\mathrm{C}^{23)}$ removal function and a CoQ10 redox cycle ${ }^{17,24)}$ defense mechanism to combat continuous exposure to oxidative stress. In the present study, along with a significant extension of running time, the group consuming a high amount of $\mathrm{QH}$ showed a more significant increase in d-ROM test value and, values of

Table 1. Changes in running times

\begin{tabular}{clcc}
\hline & \multicolumn{2}{c}{ Running time (min) } & \\
\cline { 2 - 3 } & Before intake & After intake & average changes \\
\hline High & $21.6 \pm 5.5$ & $36.0 \pm 28.4^{\mathrm{a}}$ & $14.4 \pm 25.8$ \\
Low & $18.8 \pm 6.3$ & $28.3 \pm 29.0^{\mathrm{a}, \mathrm{b}}$ & $9.4 \pm 23.7$ \\
\hline
\end{tabular}

High, $300 \mathrm{mg} / \mathrm{Kg}$ QH consumption group (n=12); Low, $30 \mathrm{mg} / \mathrm{Kg}$ $\mathrm{QH}$ consumption group $(\mathrm{n}=11){ }^{\mathrm{a}}$ : significant by Mann-Whitney $\mathrm{U}$ test compared to Before intake $(\mathrm{p}<0.05)$, : significant by Mann-Whitney U test compared to High $(\mathrm{p}<0.05)$

Table 2. Changes in oxidative stress

\begin{tabular}{ccccccc}
\hline & \multicolumn{2}{c}{ d-ROM test $($ U.CARR) } & \multicolumn{2}{c}{ BAP test $(\mu \mathrm{M})$} & \multicolumn{2}{c}{ BAP/d-ROM ratio } \\
\cline { 2 - 7 } & Pre & Post & Pre & Post & Pre & Post \\
\hline High & $113.8 \pm 4.4$ & $132.3 \pm 15.4^{\mathrm{a}}$ & $2223.2 \pm 135.2$ & $3296.7 \pm 348.2^{\mathrm{a}}$ & $19.587 \pm 1.693$ & $25.289 \pm 4.230^{\mathrm{a}}$ \\
Low & $114.7 \pm 7.7$ & $120.5 \pm 7.7^{\mathrm{b}}$ & $2202.5 \pm 58.9$ & $3317.6 \pm 260.1^{\mathrm{a}}$ & $19.275 \pm 1.273$ & $27.556 \pm 1.894^{\mathrm{a}}$ \\
\hline
\end{tabular}

High, $300 \mathrm{mg} / \mathrm{Kg} \mathrm{QH}$ consumption group (n=12); Low, $30 \mathrm{mg} / \mathrm{Kg} \mathrm{QH}$ consumption group (n=11); d-ROM test, reactive oxygen metabolites test; BAP test, biological antioxidant potential; BAP/d-ROM ratio, BAP test/d-ROM test; Pre, before QH consumption; Post, after QH consumption. ${ }^{a}$ : significant by Mann-Whitney U test compared to Pre $(\mathrm{p}<0.01),{ }^{\text {b}}$ : significant by Mann-Whitney U test compared to High $(\mathrm{p}<0.05)$

Table 3. Changes in plasma concentration

\begin{tabular}{ccccccc}
\hline & \multicolumn{2}{c}{ Plasma QH $(\mu \mathrm{g} / \mathrm{mL})$} & \multicolumn{2}{c}{ Plasma Q10 $(\mu \mathrm{g} / \mathrm{mL})$} & \multicolumn{2}{c}{ Reduced ratio } \\
\cline { 2 - 7 } & Pre & Post & Pre & Post & Pre & Post \\
\hline High & $0.031 \pm 0.021$ & $0.318 \pm 0.145^{\mathrm{a}}$ & $0.024 \pm 0.006$ & $0.065 \pm 0.073^{\mathrm{b}}$ & $0.544 \pm 0.078$ & $0.824 \pm 0.059^{\mathrm{a}}$ \\
Low & $0.033 \pm 0.023$ & $0.033 \pm 0.006^{\mathrm{d}}$ & $0.030 \pm 0.014$ & $0.018 \pm 0.006^{\mathrm{c}, \mathrm{d}}$ & $0.507 \pm 0.116$ & $0.651 \pm 0.060^{\mathrm{b}, \mathrm{d}}$ \\
\hline
\end{tabular}

High, $300 \mathrm{mg} / \mathrm{Kg} \mathrm{QH}$ consumption group ( $\mathrm{n}=12$ ); Low, $30 \mathrm{mg} / \mathrm{Kg} \mathrm{QH}$ consumption group (n=11); plasma QH, plasma concentration of QH; plasma Q10, plasma concentration of ubiquinone; reduced ratio, calculated from plasma QH and plasma Q10; Pre, before QH consumption; Post, after QH consumption. ${ }^{a}$ : significant by Mann-Whitney U test compared to Pre ( $\left.\mathrm{p}<0.001\right),{ }^{\mathrm{b}}$ : significant by Mann-Whitney U test compared to Pre $(p<0.01)$, ${ }^{c}$ : significant by Mann-Whitney U test compared to Pre $(p<0.05)$, ${ }^{d}$ significant by Mann-Whitney U test compared to High $(\mathrm{p}<0.001)$ 
plasma QH and plasma CoQ10 and a reduced ratio compared with the group consuming a low amount. This suggests the possibility that the CoQ10 redox cycle defense mechanism was activated as a result of increased oxidative stress by the extension of running time in the group consuming a high amount of QH. This CoQ10 redox cycle is a mechanism that maintains $\mathrm{QH}$ in the body by reducing enzymes and one of the important defense mechanisms that reduces exerciseinduced oxidized stress occurring with exercise. In this study, both groups showed a significant increase in BAP test value and $\mathrm{BAP} / \mathrm{d}-\mathrm{ROM}$ ratio as a result of $\mathrm{QH}$ consumption. Considering BAP test analyses, reducing the capacity of iron ions by plasma, the possibility was suggested that it might reflect improvement of the systemic anti-oxidant state rather than the effect of different amounts of consumed $\mathrm{QH}$ on plasma $\mathrm{QH}$ value. Therefore, it is necessary to study the plasma vitamin $\mathrm{C}$ level in the future. Deguchi et al. ${ }^{25)}$ reported improvement of the reduced ratio, fatigue and QOL in elderly people as a result of $\mathrm{QH}$ consumption. The reduced ratio is largely influenced by the decrease in plasma $\mathrm{QH}$ value caused by oxidative stress (or an increase in the plasma Q10 value) and the amount of NAD (P) H necessary for re-reduction of oxidized CoQ10. Thus, $\mathrm{QH}$ consumption improved the physical energy state by activating energy production, which led to improvement of the reduced ratio and fatigue. In the present study, the group consuming a high amount of QH showed a significant increase in plasma QH value and reduced ratio. The increase in plasma $\mathrm{QH}$ value caused a change in the dynamic state of $\mathrm{QH}$ in the body, suggesting the activation of the reduced enzyme system. The group consuming a low amount, however, did not show a change in plasma $\mathrm{QH}$ value, but a change was seen in the reduced ratio and running time. No change was observed in the plasma $\mathrm{QH}$ value this time, possibly because orally administered QH moved swiftly to organs and showed no apparent change. It was considered necessary to examine the levels of QH in organs in the future.

\section{REFERENCES}

1) Nagata K, Hasegawa T, Hirakado $Y$, et al.: Lifestyle-related diseases and the oxidative stress regulation system. Jap PsychoSocial Med, 2008, 48: 177-183 (in Japanese)

2) Ji LL: Oxidative stress during exercise; Implication of antioxidant nutrients. Free Radic Biol Med, 1995, 18: 1079-1086. [Medline] [CrossRef]

3) Sen CK: Oxidants and antioxidants in exercise. J Appl Physio, 1995, 179: 675-686.

4) Liu J, Yeo HC, Övervik-Douki E, et al.: Chronically and acutely exercised rats: biomarkers of oxidative stress and endogenous antioxidants. J Appl Physiol, 2000, 89: 21-28. [Medline]

5) Furukura S, Yosikawa T: Classification (Meal medicine) of health food and drugs, clinical trial, Rinn Yakuri, 2006: 37, 111-114 (in Japanese).

6) Maruoka H, Fujii K: Effect of the reduced coenzyme Q10 and exercise training on the oxidative stress regulation system and exercise capacity in mice. Jap J Complementary Alternative Med. 2011: 8, 85-97 (in Japanese). [CrossRef]

7) Abe K: Body movement and metabolism, function system of the coenzyme Q. Vitamin, 2004, 78: 283-286 (in Japanese).

8) Wada H, Goto H: Redox status of coenzyme Q10 is associated with chronological age. J Am Geriatr Soc, 2007: 55, 1141-1142. [Medline] [CrossRef]

9) Yan J, Fujii K, Yao J, et al.: Reduced coenzyme Q10 supplementation decelerates senescence in SAMP1 mice. Exp Gerontol, 2006, 41: 130-140. [Medline] [CrossRef]

10) Hosokawa M: Grading score system, A method for evaluation of the degree of senescence in senescence accelerated mouse (SAM). Mech Ageing Dev, 1984: 26, 91-102. [Medline] [CrossRef]

11) Brooks GA, White TP: Determination of metabolic and heart rate responses of rats to treadmill exercise. J Appl Physiol, 1978, 45: 1009-1015. [Medline]

12) Jan Vitamin Society. In: encyclopedia of the Vitamin 4.ubiquinone. Tokyo: Asakurasyoten, 1996, pp 483-496 (in Japanese).

13) Hosoe K, Kitano M, Kishida H, et al.: Study on safety and bioavailability of ubiquinol (Kaneka QH) after single and 4-week multiple oral administration to healthy volunteers. Regul Toxicol Pharmacol, 2007: 47, 19-28. [Medline] [CrossRef]

14) Alberti A, Bolognini L, Macciantelli $\mathrm{D}$, et al.: The radical cation of $\mathrm{N}$, $\mathrm{N}$-diethyl-para-phenylendiamine; a possible indicator of oxidative stress in biological samples. Res Chem Intermed, 2000: 26, 253-267. [CrossRef]

15) Iamele L, Fiocchi R, Vernocchi A: Evaluation of an automated spectrophotometric assay for reactive oxygen metabolites in serum. Clin Chem Lab Med, 2002: 40, 673-676. [Medline] [CrossRef]

16) Kishi T, Okamoto T: Cardiostimulatory action of coenzyme $Q$ homologues an cultured myocardial cells and their biochemical mechanisms. Clin Investig, 1993, 71, S71-S75. [Medline]

17) Bowry VW, Stanley KK, Stocker R: High density lipoprotein is the major carrier of lipid hydroperoxides in human blood plasma from fasting donors. Proc Natl Acad Sci, 1992, 89: 10316-10320. [Medline] [CrossRef]

18) Saito Y: Kangengata coenzyme Q10 no subete. Tokyo: Ark Shuppan, 2010, pp 14-75 (in Japanese).

19) Mizuno K, Tanaka M, Nozaki S, et al.: Antifatigue effects of coenzyme Q10 during physical fatigue. Nutrition, 2008: 24, 293-299. [Medline] [CrossRef]

20) Langsjoen PH, Langsjoen AM: Supplemental ubiquinol in patients with advanced congestive heart failure. BioFactors, 2008: 32, 119-128. [Medline] [CrossRef]

21) Goto $S$, Radak $Z$, Nyakas $C$, et al.: Regular exercise; an effective means to reduce oxidative stress in old rats. Ann N Y Acad Sci, 2004, 1019: 471-474. [Medline] [CrossRef]

22) Kimura N, Higuthi M: The problem of exercise and senescence, oxidative stress.Rinn Sports Igaku, 2006: 23, 33-39 (in Japanese).

23) Ishigami A: Anti-aging research using SMP30/GNL knockout mice. Yakugaku Zasshi, 2010, 130: 25-28 (in Japanese). [Medline] [CrossRef]

24) Ji LL: Antioxidant enzyme response to exercise and aging. Med Sci Sports Exerc, 1993: 25, 225-231. [Medline]

25) Deguchi S, Fujii K, Kurihara T: The effect of the reduced form of coenzyme Q10 (ubiquinol, Kaneka $\mathrm{QH}^{\mathrm{TM}}$ ) on QOL improvement in the elderly. Rinsyo Iyaku, 2008, 24: 233-238 (in Japanese). 